\title{
On coefficient problems for functions starlike with respect to symmetric points
}

\section{Paweł Zaprawa ${ }^{1}$ (D)}

Received: 19 November 2021 / Accepted: 4 January 2022 / Published online: 20 January 2022

(C) The Author(s) 2022

\begin{abstract}
The main idea of the study on coefficient problems in various classes of analytic functions (univalent or nonunivalent) is to express the coefficients of functions in a given class by the coefficients of corresponding functions with positive real part. Thus, coefficient functionals can be studied using inequalities known for the class $\mathcal{P}$. Lemmas obtained by Libera and Złotkiewicz and by Prokhorov and Szynal play a special role in this approach. Recently, a new way leading to results on coefficient functionals has been pointed out. This approach is based on relating the coefficients of functions in a given class and the coefficients of corresponding Schwarz functions. In many cases, if we follow this approach, it is easy to predict the exact estimate of the functional and make the appropriate computations. In the proofs of these estimates are used not only classical results (the Schwarz-Pick Lemma or Wiener's inequality), but also inequalities obtained either recently (e.g. by Efraimidis) or long ago yet almost forgotten (Carlson's inequality). In this paper, a number of coefficient problems will be solved using the new approach described above. The object of our study is the class of starlike functions with respect to symmetric points associated with the exponential function.
\end{abstract}

Keywords Coefficient problems $\cdot$ Logarithmic coefficients $\cdot$ Zalcman functionals $\cdot$ Hankel determinants $\cdot$ Starlike functions

Mathematics Subject Classification 30C50 - 30C45

Paweł Zaprawa

p.zaprawa@pollub.pl

1 Department of Mathematics, Faculty of Mechanical Engineering, Lublin University of Technology, Nadbystrzycka 36, 20-618 Lublin, Poland 


\section{Introduction}

Let $\mathbb{D}$ be the unit disk $\{z \in \mathbb{C}:|z|<1\}$ and $\mathcal{A}$ be the family of all functions $f$ analytic in $\mathbb{D}$, normalized by the condition $f(0)=f^{\prime}(0)-1=0$. It means that $f$ has the expansion

$$
f(z)=z+\sum_{n=2}^{\infty} a_{n} z^{n} .
$$

Let also $\mathcal{B}_{0}$ be the class of Schwarz functions, i.e., analytic functions $\omega: \mathbb{D} \rightarrow \mathbb{D}$, $\omega(0)=0$. The function $\omega \in \mathcal{B}_{0}$ can be written as a power series

$$
\omega(z)=\sum_{n=1}^{\infty} c_{n} z^{n}
$$

For given analytic functions $f$ and $g$ in $\mathbb{D}$, we say that $f$ is subordinate to $g$ in $\mathbb{D}$ and write $f \prec g$ if there exists $\omega \in \mathcal{B}_{0}$ such that

$$
f(z)=g(\omega(z)), \quad z \in \mathbb{D} .
$$

Moreover, if the function $g$ is univalent in $\mathbb{D}$, then $f \prec g$ if and only if $f(0)=g(0)$ and $f(\mathbb{D}) \subset g(\mathbb{D})$.

Let $\mathcal{S}_{S}^{*}$ denote the class of functions which are starlike with respect to the symmetric points. A function $f$ is in $\mathcal{S}_{S}^{*}$ if

$$
\frac{2 z f^{\prime}(z)}{f(z)-f(-z)} \prec \varphi_{0}(z), \quad z \in \mathbb{D}
$$

where

$$
\varphi_{0}(z)=\frac{1+z}{1-z} .
$$

If the function $\varphi_{0}(z)$ is replaced by any analytic univalent function $\varphi$ with positive real part in $\mathbb{D}$ and symmetric with respect to the real axis, then we obtain the class $\mathcal{S}_{S}^{*}(\varphi(z))$.

In this paper, we consider the class $\mathcal{S}_{S}^{*}(\varphi(z))$ with $\varphi(z)=e^{z}$. Hence, we can write

$$
\mathcal{S}_{S}^{*}\left(e^{z}\right)=\left\{f \in \mathcal{S}: \frac{2 z f^{\prime}(z)}{f(z)-f(-z)}=e^{\omega(z)}, \quad \omega \in \mathcal{B}_{0}, z \in \mathbb{D}\right\} .
$$

This class was first discussed by Ganesh et al. in [3], where some coefficients functionals were estimated. The majority of results were not sharp. The main tool used to obtain those results was a lemma proved by Libera and Złotkiewicz.

In this paper, we follow a new approach which is based on relating the coefficients of functions in a given class and the coefficients of corresponding Schwarz functions. In many cases, it is easy to predict the exact estimate of the functional and make the appropriate computations. It is the case for the class $\mathcal{S}_{S}^{*}\left(e^{z}\right)$. By applying the lemmas proved by Libera and Złotkiewicz and by Prokhorov and 
Szynal as well as some other tools, and by performing the calculus more precisely, we are able to derive better estimates, almost all of them being sharp.

To prove our results, we need the following lemmas for Schwarz functions. The first one is the above-mentioned result obtained by Prokhorov and Szynal.

Lemma 1 ([5]) Let $\omega(z)=c_{1} z+c_{2} z^{2}+\cdots$ be a Schwarz function. Then, for any real numbers $\mu$ and $v$ such that

$$
(\mu, v) \in\left\{|\mu| \leq \frac{1}{2},-1 \leq v \leq 1\right\} \cup\left\{\frac{1}{2} \leq|\mu| \leq 2, \frac{4}{27}(|\mu|+1)^{3}-(|\mu|+1) \leq v \leq 1\right\},
$$

the following sharp estimate holds

$$
\left|c_{3}+\mu c_{1} c_{2}+v c_{1}^{3}\right| \leq 1
$$

From the Schwarz-Pick Lemma, it follows that for $\omega \in \mathcal{B}_{0}$ of the form (1.2),

$$
\left|c_{2}\right| \leq 1-\left|c_{1}\right|^{2} \text {. }
$$

This inequality can be improved (see, for example, [2]) as follows. For any $\lambda \in \mathbb{C}$,

$$
\left|c_{2}+\lambda c_{1}^{2}\right| \leq \max \{1,|\lambda|\}
$$

Carlson in [1] obtained another generalization of the Schwarz-Pick Lemma. Here, we state only these inequalities which are useful for our purpose (for all details, see [7]).

Lemma 2 ( [1]) Let $\omega(z)=c_{1} z+c_{2} z^{2}+\cdots$ be a Schwarz function. Then,

$$
\begin{gathered}
\left|c_{3}\right| \leq 1-\left|c_{1}\right|^{2}-\frac{\left|c_{2}\right|^{2}}{1+\left|c_{1}\right|} \quad, \quad\left|c_{4}\right| \leq 1-\left|c_{1}\right|^{2}-\left|c_{2}\right|^{2}, \\
\left|c_{5}\right| \leq 1-\left|c_{1}\right|^{2}-\left|c_{2}\right|^{2}-\frac{\left|c_{3}\right|^{2}}{1+\left|c_{1}\right|} .
\end{gathered}
$$

The above lemma immediately results in the following fact.

Lemma 3 Let $\omega(z)=c_{1} z+c_{2} z^{2}+\cdots$ be a Schwarz function. Then

$$
\left|c_{1} c_{3}-c_{2}^{2}\right| \leq 1-\left|c_{1}\right|^{2} \text {. }
$$

We also need the results obtained by Efraimidis.

Lemma 4 ([2]) Let $\omega(z)=c_{1} z+c_{2} z^{2}+\cdots$ be a Schwarz function and $\lambda \in \mathbb{C}$. Then

$$
\left|c_{4}+(1+\lambda) c_{1} c_{3}+c_{2}^{2}+(1+2 \lambda) c_{1}^{2} c_{2}+\lambda c_{1}^{4}\right| \leq \max \{1,|\lambda|\}
$$

and 


$$
\left|c_{4}+2 c_{1} c_{3}+\lambda c_{2}^{2}+(1+2 \lambda) c_{1}^{2} c_{2}+\lambda c_{1}^{4}\right| \leq \max \{1,|\lambda|\} .
$$

The method used by Efraimidis in the proof of his lemma has much greater potential. Based on this method, we can obtain some inequalities involving the fifth coefficient of $\omega \in \mathcal{B}_{0}$ (see also [6]).

Lemma 5 If $\omega \in \mathcal{B}_{0}$ is of the form (1.2) and $\mu \in \mathbb{C},|\mu| \leq 1$, then

$$
\begin{gathered}
\mid c_{5}+(1+\mu) c_{1} c_{4}+(1+\mu) c_{2} c_{3}+3 \mu c_{1} c_{2}^{2}+\left(1+\mu+\mu^{2}\right) c_{1}^{2} c_{3} \\
+2 \mu(1+\mu) c_{1}^{3} c_{2}+\mu^{2} c_{1}^{5} \mid \leq 1 .
\end{gathered}
$$

\section{Coefficient bounds}

We start with the coefficients of $f \in \mathcal{S}_{S}^{*}\left(e^{z}\right)$. Applying in

$$
\frac{2 z f^{\prime}(z)}{f(z)-f(-z)}=e^{\omega(z)}
$$

the expansions of $f$ and $\omega$ given by (1.1) and (1.2), we obtain

$$
\begin{aligned}
& a_{2}=\frac{1}{2} c_{1} \\
& a_{3}=\frac{1}{2} c_{2}+\frac{1}{4} c_{1}^{2} \\
& a_{4}=\frac{1}{4} c_{3}+\frac{3}{8} c_{1} c_{2}+\frac{5}{48} c_{1}^{3} \\
& a_{5}=\frac{1}{4} c_{4}+\frac{1}{4} c_{1} c_{3}+\frac{1}{4} c_{2}^{2}+\frac{1}{4} c_{1}^{2} c_{2}+\frac{1}{24} c_{1}^{4} \\
& a_{6}=\frac{1}{6} c_{5}+\frac{5}{24} c_{1} c_{4}+\frac{1}{4} c_{2} c_{3}+\frac{5}{24} c_{1} c_{2}^{2}+\frac{1}{6} c_{1}^{2} c_{3}+\frac{1}{8} c_{1}^{3} c_{2}+\frac{11}{720} c_{1}^{5} .
\end{aligned}
$$

From [3], it is known that if $f \in \mathcal{S}_{S}^{*}\left(e^{z}\right)$ is of the form (1.1), then $\left|a_{2}\right| \leq \frac{1}{2}$ and $\left|a_{3}\right| \leq \frac{1}{2}$.

Theorem 1 If $f \in \mathcal{S}_{S}^{*}\left(e^{z}\right)$ is of the form (1.1), then

$$
\left|a_{4}\right| \leq \frac{1}{4} \quad \text { and } \quad\left|a_{5}\right| \leq \frac{1}{4} .
$$

The bounds are sharp.

Proof Lemma 1 with $\mu=\frac{3}{2}$ and $v=\frac{5}{12}$ applied to

$$
\left|a_{4}\right|=\frac{1}{4}\left|c_{3}+\frac{3}{2} c_{1} c_{2}+\frac{5}{12} c_{1}^{3}\right|,
$$

results in the first inequality.

To prove the second inequality, we can write 


$$
4\left|a_{5}\right|=\left|\frac{1}{2}\left(c_{4}+2 c_{1} c_{3}+c_{2}^{2}+3 c_{1}^{2} c_{2}+c_{1}^{4}\right)+\frac{1}{2}\left(c_{4}+c_{2}^{2}-c_{1}^{2} c_{2}-\frac{2}{3} c_{1}^{4}\right)\right| .
$$

From (1.4) with $\lambda=1$, the first component is bounded by $1 / 2$. By Lemma 2 , the second component, can be estimated as follows

$$
\begin{aligned}
\frac{1}{2}\left|c_{4}+c_{2}^{2}-c_{1}^{2} c_{2}-\frac{2}{3} c_{1}^{4}\right| & \leq \frac{1}{2}\left[1-\left|c_{1}\right|^{2}-\left|c_{2}\right|^{2}+\left|c_{2}\right|^{2}+\left|c_{1}\right|^{2}\left(1-\left|c_{1}\right|^{2}\right)+\frac{2}{3}\left|c_{1}\right|^{4}\right] \\
& =\frac{1}{2}-\frac{1}{6}\left|c_{1}\right|^{4} \leq \frac{1}{2} .
\end{aligned}
$$

Combining the estimates of both components of (2.3), we get $\left|a_{5}\right| \leq \frac{1}{4}$.

If $c_{3}=1$ and $c_{k}=0$ for $k \neq 3$, then $a_{4}=\frac{1}{4}$. Similarly, if $c_{4}=1$ and $c_{k}=0$ for $k \neq 4$, then $a_{5}=\frac{1}{4}$. This means that the equalities in the assertion of this theorem hold for the functions given by (2.1) with $\omega(z)=z^{3}$ and $\omega(z)=z^{4}$, respectively.

The logarithmic coefficients of a given univalent function $f$, denoted by $\gamma_{n}=\gamma_{n}(f)$, are defined as

$$
\frac{1}{2} \log \left(\frac{f(z)}{z}\right)=\sum_{n=1}^{\infty} \gamma_{n} z^{n} .
$$

If $f$ is given by (1.1), then its logarithmic coefficients are given as follows

$$
\begin{aligned}
& \gamma_{1}=\frac{1}{2} a_{2} \\
& \gamma_{2}=\frac{1}{2}\left(a_{3}-\frac{1}{2} a_{2}^{2}\right) \\
& \gamma_{3}=\frac{1}{2}\left(a_{4}-a_{2} a_{3}+\frac{1}{3} a_{2}^{3}\right) \\
& \gamma_{4}=\frac{1}{2}\left(a_{5}-a_{2} a_{4}+a_{2}^{2} a_{3}-\frac{1}{2} a_{3}^{2}-\frac{1}{4} a_{2}^{4}\right) \\
& \gamma_{5}=\frac{1}{2}\left(a_{6}-a_{2} a_{5}-a_{3} a_{4}+a_{2} a_{3}^{2}+a_{2}^{2} a_{4}-a_{2}^{3} a_{3}+\frac{1}{5} a_{2}^{5}\right) .
\end{aligned}
$$

The sharp bounds of $\gamma_{k}, k=1,2,3,4,5$ are established in the next theorem.

Theorem 2 If $f \in \mathcal{S}_{S}^{*}\left(e^{z}\right)$ is of the form (1.1), then

$$
\left|\gamma_{1}\right| \leq \frac{1}{4} \quad, \quad\left|\gamma_{2}\right| \leq \frac{1}{4} \quad, \quad\left|\gamma_{3}\right| \leq \frac{1}{8} \quad, \quad\left|\gamma_{4}\right| \leq \frac{1}{8} \quad, \quad\left|\gamma_{5}\right| \leq \frac{1}{12}
$$

The bounds are sharp.

Proof Applying (2.2) in (2.5), we have 


$$
\begin{aligned}
& \gamma_{1}=\frac{1}{4} c_{1} \\
& \gamma_{2}=\frac{1}{4}\left(c_{2}+\frac{1}{4} c_{1}^{2}\right) \\
& \gamma_{3}=\frac{1}{8}\left(c_{3}+\frac{1}{2} c_{1} c_{2}+\frac{1}{12} c_{1}^{3}\right) \\
& \gamma_{4}=\frac{1}{8}\left(c_{4}+\frac{1}{2} c_{1} c_{3}+\frac{1}{2} c_{2}^{2}+\frac{1}{4} c_{1}^{2} c_{2}+\frac{1}{48} c_{1}^{4}\right) \\
& \gamma_{5}=\frac{1}{12}\left(c_{5}+\frac{1}{2} c_{1} c_{4}+\frac{3}{4} c_{2} c_{3}+\frac{1}{16} c_{1}^{3} c_{2}+\frac{1}{4} c_{1}^{2} c_{3}+\frac{1}{8} c_{1} c_{2}^{2}+\frac{1}{240} c_{1}^{5}\right) .
\end{aligned}
$$

The bounds of $\gamma_{1}$ and $\gamma_{2}$ are clear. The result for $\gamma_{3}$ immediately follows from Lemma 1 with $\mu=\frac{1}{2}$ and $v=\frac{1}{12}$.

Observe that

$$
\begin{aligned}
\mid c_{4} & +\frac{1}{2} c_{1} c_{3}+\frac{1}{2} c_{2}^{2}+\frac{1}{4} c_{1}^{2} c_{2}+\frac{1}{48} c_{1}^{4} \mid \\
& =\left|\frac{1}{2}\left(c_{4}+c_{1} c_{3}+c_{2}^{2}+c_{1}^{2} c_{2}\right)+\frac{1}{2}\left(c_{4}-\frac{1}{2} c_{1}^{2} c_{2}+\frac{1}{24} c_{1}^{4}\right)\right| \leq 1 .
\end{aligned}
$$

Indeed, from (1.4) with $\lambda=0$,

$$
\frac{1}{2}\left|c_{4}+c_{1} c_{3}+c_{2}^{2}+c_{1}^{2} c_{2}\right| \leq \frac{1}{2}
$$

and, by Lemma 2 and (1.3),

$$
\left|\frac{1}{2} c_{4}-\frac{1}{4} c_{1}^{2} c_{2}+\frac{1}{48} c_{1}^{4}\right| \leq \frac{1}{2}\left[1-\left|c_{1}\right|^{2}-\left|c_{2}\right|^{2}+\frac{1}{2}\left|c_{1}\right|^{2}\left(1-\left|c_{1}\right|^{2}\right)+\frac{1}{24}\left|c_{1}\right|^{4}\right],
$$

which is clearly less than or equal to $1 / 2$.

For $\gamma_{5}$, we have

$$
\begin{aligned}
12\left|\gamma_{5}\right|= & \frac{1}{2} \mid\left(c_{5}+c_{1} c_{4}+c_{2} c_{3}+c_{1}^{2} c_{3}\right) \\
& +\left(c_{5}+\frac{1}{2} c_{2} c_{3}-\frac{1}{2} c_{1}^{2} c_{3}+\frac{1}{8} c_{1}^{3} c_{2}+\frac{1}{4} c_{1} c_{2}^{2}+\frac{1}{120} c_{1}^{5}\right) \mid \leq \frac{1}{2}(1+W) .
\end{aligned}
$$

The bound of the first component follows from (1.6) with $\mu=0$. The triangle inequality and Lemma 2 result in

$$
\begin{aligned}
W \leq & 1-\left|c_{1}\right|^{2}-\left|c_{2}\right|^{2}-\frac{\left|c_{3}\right|^{2}}{1+\left|c_{1}\right|}+\frac{1}{2}\left(\left|c_{2}\right|+\left|c_{1}\right|^{2}\right)\left|c_{3}\right| \\
& +\frac{1}{8}\left|c_{1}\right|^{3}\left|c_{2}\right|+\frac{1}{4}\left|c_{1}\right|\left|c_{2}\right|^{2}+\frac{1}{120}\left|c_{1}\right|^{5} .
\end{aligned}
$$

The above expression takes its greatest value with respect to $\left|c_{3}\right|$ when $\left|c_{3}\right|=\frac{1}{4}\left(\left|c_{2}\right|+\left|c_{1}\right|^{2}\right)\left(1+\left|c_{1}\right|\right)$, so

$$
\begin{aligned}
W \leq & 1-\left|c_{1}\right|^{2}+\frac{1}{16}\left|c_{1}\right|^{4}+\frac{17}{240}\left|c_{1}\right|^{5}-\frac{15}{16}\left|c_{2}\right|^{2}+\frac{5}{16}\left|c_{1}\right|\left|c_{2}\right|^{2} \\
& +\frac{1}{8}\left|c_{1}\right|^{2}\left|c_{2}\right|+\frac{1}{4}\left|c_{1}\right|^{3}\left|c_{2}\right| .
\end{aligned}
$$

Since $\left|c_{1}\right|^{3}\left|c_{2}\right| \leq\left|c_{1}\right|^{2}\left|c_{2}\right|$, we can write 


$$
W \leq h(c, d)
$$

where $c=\left|c_{1}\right|, d=\left|c_{2}\right|$ and

$$
h(c, d)=1-c^{2}+\frac{1}{16} c^{4}+\frac{17}{240} c^{5}-\frac{15}{16} d^{2}+\frac{5}{16} c d^{2}+\frac{3}{8} c^{2} d .
$$

The shape of the region of variability of $(c, d)$ is a simple consequence of the Schwarz-Pick Lemma. It coincides with

$$
\Omega=\left\{(c, d): 0 \leq c \leq 1,0 \leq d \leq 1-c^{2}\right\} .
$$

It is not difficult to show that $(0,0)$ is the only critical point of $h$ in $\Omega$. Consequently, it is enough to derive the greatest value of $h$ on the boundary of $\Omega$. But,

$$
\begin{aligned}
h(c, 0) & =1-c^{2}+\frac{1}{16} c^{4}+\frac{17}{240} c^{5} \leq 1, \\
h(0, d) & =1-\frac{15}{16} d^{2} \leq 1, \\
h\left(c, 1-c^{2}\right) & =\frac{1}{16}+\frac{5}{16} c+\frac{5}{4} c^{2}-\frac{5}{8} c^{3}-\frac{5}{4} c^{4}+\frac{23}{60} c^{5} .
\end{aligned}
$$

Since $\frac{5}{4}\left(c^{2}-c^{4}\right) \leq \frac{5}{16}$ and $\frac{5}{16}\left(c-2 c^{3}+c^{5}\right) \leq \frac{\sqrt{5}}{25}$ we conclude that

$$
h\left(c, 1-c^{2}\right) \leq \frac{1}{16}+\frac{5}{16}+\frac{\sqrt{5}}{25}+\left(\frac{23}{60}-\frac{5}{16}\right) c^{5}<1 .
$$

Combining all these inequalities, we get

$$
h(c, d) \leq h(0,0)=1,
$$

which results in the desired bound.

Observe that we obtain equalities in each bound of $\gamma_{k}, k=1,2,3,4,5$ when $\omega(z)=z^{k}$. This means that the obtained estimates are sharp.

\section{Estimates of Zalcman functionals and Hankel determinants}

It is known ([3]) that if $f \in \mathcal{S}_{S}^{*}\left(e^{z}\right)$ is of the form (1.1), then $\left|a_{3}-a_{2}^{2}\right| \leq \frac{1}{2}$. This functional, known as the Fekete-Szegö functional, is a particular case of the Zalcman functional $a_{n+m-1}-a_{n} a_{m}$. Let us consider other cases of the Zalcman functional.

The estimate of $a_{4}-a_{2} a_{3}$ is a simple consequence of Lemma 1. Namely,

$$
\left|a_{4}-a_{2} a_{3}\right|=\frac{1}{4}\left|c_{3}+\frac{1}{2} c_{1} c_{2}-\frac{1}{12} c_{1}^{3}\right|,
$$

so taking $\mu=\frac{1}{2}$ and $v=-\frac{1}{12}$ in Lemma 1 yields

$$
\left|a_{4}-a_{2} a_{3}\right| \leq \frac{1}{4}
$$

To estimate $a_{5}-a_{3}^{2}$, we write this expression as follows 


$$
\begin{aligned}
\left|a_{5}-a_{3}^{2}\right| & =\frac{1}{4}\left|c_{4}+c_{1} c_{3}-\frac{1}{12} c_{1}^{4}\right| \\
& =\frac{1}{4}\left|\frac{1}{2}\left(c_{4}+2 c_{1} c_{3}-\frac{1}{2} c_{2}^{2}-\frac{1}{2} c_{1}^{4}\right)+\frac{1}{2}\left(c_{4}+\frac{1}{2} c_{2}^{2}+\frac{1}{3} c_{1}^{4}\right)\right| .
\end{aligned}
$$

Applying (1.5) with $\lambda=-\frac{1}{2}$, the first component is bounded by $1 / 2$. To estimate the other, we use the triangle inequality and Lemma 2 . Hence

$$
\begin{aligned}
\frac{1}{2}\left|c_{4}+\frac{1}{2} c_{2}^{2}+\frac{1}{3} c_{1}^{4}\right| & \leq \frac{1}{2}\left(1-\left|c_{1}\right|^{2}-\left|c_{2}\right|^{2}+\frac{1}{2}\left|c_{2}\right|^{2}+\frac{1}{3}\left|c_{1}\right|^{4}\right) \\
& \leq \frac{1}{2}\left[1-\frac{1}{3}\left|c_{1}\right|^{2}\left(3-\left|c_{1}\right|^{2}\right)-\frac{1}{2}\left|c_{2}\right|^{2}\right] \leq \frac{1}{2}
\end{aligned}
$$

so

$$
\left|a_{5}-a_{3}^{2}\right| \leq \frac{1}{4}
$$

Considering $\omega(z)=z^{3}$ and $\omega(z)=z^{4}$, we can observe that the estimates of the two cases of the Zalcman functional are sharp. We have proved what follows.

Theorem 3 If $f \in \mathcal{S}_{S}^{*}\left(e^{z}\right)$ is of the form (1.1), then the following sharp bounds hold

$$
\left|a_{4}-a_{2} a_{3}\right| \leq \frac{1}{4} \quad \text { and } \quad\left|a_{5}-a_{3}^{2}\right| \leq \frac{1}{4} .
$$

Let us turn to Hankel determinants for the class $\mathcal{S}_{S}^{*}\left(e^{z}\right)$. The first result is easy to obtain.

Theorem 4 If $f \in \mathcal{S}_{S}^{*}\left(e^{z}\right)$ is of the form (1.1), then

$$
\left|H_{2,2}\right| \leq \frac{1}{4} \text {. }
$$

Proof If $f \in \mathcal{S}_{S}^{*}\left(e^{z}\right)$, then

$$
\left|H_{2,2}\right|=\left|a_{2} a_{4}-a_{3}^{2}\right|=\frac{1}{4}\left|\frac{1}{2}\left(c_{2}^{2}-c_{1} c_{3}\right)+\frac{1}{2}\left(c_{2}^{2}+\frac{1}{2} c_{1}^{2} c_{2}+\frac{1}{12} c_{1}^{4}\right)\right| .
$$

Since

$$
\left|c_{2}^{2}+\frac{1}{2} c_{1}^{2} c_{2}+\frac{1}{12} c_{1}^{4}\right| \leq\left(1-\left|c_{1}\right|^{2}\right)^{2}+\frac{1}{2} c_{1}^{2}\left(1-\left|c_{1}\right|^{2}\right)+\frac{1}{12} c_{1}^{4}=1-\frac{3}{2}\left|c_{1}\right|^{2}+\frac{7}{12}\left|c_{1}\right|^{4}
$$

and

$$
[0,1] \ni t \rightarrow 1-\frac{3}{2} t+\frac{7}{12} t^{2}
$$

is a decreasing function, we conclude that 


$$
\left|c_{2}^{2}+\frac{1}{2} c_{1}^{2} c_{2}+\frac{1}{12} c_{1}^{4}\right| \leq 1 .
$$

This and Lemma 3 result in the declared bound of $H_{2,2}$.

Furthermore, $\left|H_{2,2}\right|=\frac{1}{4}$ if $c_{2}=1$ and $c_{k}=0$ for $k \neq 2$, i.e. if $\omega(z)=z^{2}$.

Theorem 5 If $f \in \mathcal{S}_{S}^{*}\left(e^{z}\right)$ is of the form (1.1), the following sharp bound holds

$$
\left|H_{2,3}\right| \leq \frac{1}{8} \text {. }
$$

Proof Assume that $f \in \mathcal{S}_{S}^{*}\left(e^{z}\right)$ is of the form (1.1). Then

$$
\begin{aligned}
\left|H_{2,3}\right| & =\left|a_{3} a_{5}-a_{4}^{2}\right| \\
& =\frac{1}{8}\left|\left(c_{2}+\frac{1}{2} c_{1}^{2}\right) c_{4}-\frac{1}{2} c_{3}^{2}-\frac{1}{2}\left(c_{2}-\frac{1}{6} c_{1}^{2}\right) c_{1} c_{3}+c_{2}^{3}+\frac{3}{8} c_{1}^{2} c_{2}^{2}+\frac{1}{24} c_{1}^{4} c_{2}-\frac{1}{288} c_{1}^{6}\right| .
\end{aligned}
$$

By the triangle inequality and Lemma 2,

$$
\begin{aligned}
\left|H_{2,3}\right| \leq & \frac{1}{8}\left[\left(\left|c_{2}\right|+\frac{1}{2}\left|c_{1}\right|^{2}\right)\left(1-\left|c_{1}\right|^{2}-\left|c_{2}\right|^{2}\right)+\frac{1}{2}\left(1-\left|c_{1}\right|^{2}-\frac{\left|c_{2}\right|^{2}}{1+\left|c_{1}\right|}\right)^{2}\right. \\
& +\frac{1}{2}\left(\left|c_{2}\right|+\frac{1}{6}\left|c_{1}\right|^{2}\right)\left|c_{1}\right|\left(1-\left|c_{1}\right|^{2}-\frac{\left|c_{2}\right|^{2}}{1+\left|c_{1}\right|}\right) \\
& \left.+\left|c_{2}\right|^{3}+\frac{3}{8}\left|c_{1}\right|^{2}\left|c_{2}\right|^{2}+\frac{1}{24}\left|c_{1}\right|^{4}\left|c_{2}\right|+\frac{1}{288}\left|c_{1}\right|^{6}\right] .
\end{aligned}
$$

Let $h\left(\left|c_{1}\right|,\left|c_{2}\right|\right)$ denote the right hand side of the above inequality and let $c=\left|c_{1}\right|$, $d=\left|c_{2}\right|$. Since

$$
\begin{aligned}
\frac{\partial h}{\partial d}= & \frac{1}{24(1+c)^{2}}\left[48 d^{3}-36 c(1+c) d^{2}-2(1+c)\left(24-21 c^{2}+5 c^{3}\right) d\right. \\
& \left.+\left(24+12 c-24 c^{2}-12 c^{3}+c^{4}\right)(1+c)^{2}\right]
\end{aligned}
$$

replacing $d^{2}$ by $d$ and omitting $c^{4}$ in the last component, we get

$$
\frac{\partial h}{\partial d} \geq \frac{g(c, d)}{24(1+c)^{2}}
$$

with

$$
g(c, d)=48 d^{3}-2(1+c)\left(24+18 c-21 c^{2}+5 c^{3}\right) d+12(2+c)(1-c)(1+c)^{3} .
$$

A straightforward algebraic computation shows that the critical points of $g$ satisfy 


$$
\left\{\begin{array}{c}
\left(21-3 c-24 c^{2}+10 c^{3}\right) d-3\left(5-6 c-5 c^{2}\right)(1+c)^{2}=0 \\
72 d^{2}-(1+c)\left(24+18 c-21 c^{2}+5 c^{3}\right)=0,
\end{array}\right.
$$

so in $\Omega$ given by (2.7), there is only one critical point $\left(c_{0}, d_{0}\right)$ where $c_{0}=0.345 \ldots$ and $d_{0}=0.722 \ldots$. For this point,

$$
g\left(c_{0}, d_{0}\right)=8.695 \ldots
$$

On the boundary of $\Omega$, we have

$$
\begin{gathered}
g(c, 0)=12(2+c)(1-c)(1+c)^{3} \geq g(1,0)=0, \\
g(0, d)=24\left(1-2 d+2 d^{3}\right) \geq g\left(0, \frac{\sqrt{3}}{3}\right)=\frac{8}{3}(9-4 \sqrt{3}), \\
g\left(c, 1-c^{2}\right)=2(1-c)(1+c)^{2}\left(12-24 c+3 c^{2}+19 c^{3}\right) .
\end{gathered}
$$

It is a simple task to show that $12-24 c+3 c^{2}+19 c^{3}>0$ in $[0,1]$, so

$$
g(c, d) \geq 0 \text { for }(c, d) \in \Omega
$$

This means that

$$
\frac{\partial h}{\partial d} \geq 0 \quad \text { for } \quad(c, d) \in \Omega
$$

Consequently,

$$
h(c, d) \leq h\left(c, 1-c^{2}\right)=1-\frac{5}{8} c^{2}-\frac{9}{8} c^{4}+\frac{217}{288} c^{6} .
$$

The function $h\left(c, 1-c^{2}\right)$ is decreasing for $c \in[0,1]$, so

$$
h(c, d) \leq h\left(c, 1-c^{2}\right) \leq 1 \text {. }
$$

Similarly as in Theorem 4, the equality $\left|H_{2,3}\right|=\frac{1}{8}$ holds if $c_{2}=1$ and $c_{k}=0$ for $k \neq 2$, i.e. if $\omega(z)=z^{2}$.

Theorem 6 If $f \in \mathcal{S}_{S}^{*}\left(e^{z}\right)$ is of the form (1.1), then

$$
\left|H_{3,1}\right| \leq \frac{13}{128} \text {. }
$$

Proof If $f \in \mathcal{S}_{S}^{*}\left(e^{z}\right)$, then

$$
\left|H_{3,1}\right|=\frac{1}{16}\left|2 c_{2} c_{4}-c_{3}^{2}+\left(c_{2}+\frac{1}{6} c_{1}^{2}\right) c_{1} c_{3}-\frac{1}{4} c_{1}^{2} c_{2}^{2}-\frac{1}{12} c_{1}^{4} c_{2}-\frac{1}{144} c_{1}^{6}\right| .
$$

At the beginning, it should be noted that 


$$
\left|c_{3}\left(c_{3}-c_{1} c_{2}-\frac{1}{6} c_{1}^{3}\right)\right| \leq\left|c_{3}\right|
$$

by Lemma 1 with $\mu=-1$ and $v=-\frac{1}{6}$. Under Lemma 2,

$$
\left|c_{3}\right| \leq 1-\left|c_{1}\right|^{2}-\frac{\left|c_{2}\right|^{2}}{1+\left|c_{1}\right|} \leq 1-\left|c_{1}\right|^{2}-\frac{\left|c_{2}\right|^{2}}{2} .
$$

Applying it and $\left|c_{4}\right| \leq 1-\left|c_{1}\right|^{2}-\left|c_{2}\right|^{2}$, we get

$$
\left|H_{3,1}\right| \leq \frac{1}{16} h\left(\left|c_{1}\right|,\left|c_{2}\right|\right),
$$

where

$$
h(c, d)=1-c^{2}-\frac{d^{2}}{2}+2 d\left(1-c^{2}-d^{2}\right)+\frac{1}{4} c^{2} d^{2}+\frac{1}{12} c^{4} d+\frac{1}{144} c^{6} \quad, \quad c=\left|c_{1}\right|, d=\left|c_{2}\right| .
$$

But $h$ is a decreasing function of the variable $c$; consequently,

$$
h(c, d) \leq h(0, d)=1+2 d-\frac{1}{2} d^{2}-2 d^{3} .
$$

The function $h(0, d)$ achieves its greatest value in $[0,1]$ if $d=1 / 2$, so $h(0, d) \leq \frac{13}{8}$, which completes the proof.

This result is not sharp. Based on Formula (3.1), it is expected that the sharp bound of $\left|H_{3,1}\right|$ is equal to $\frac{1}{16}$.

The method used in the proof of Theorem 2 for the bound $\left|\gamma_{5}\right| \leq \frac{1}{12}$ may be adopted to prove that if $f \in \mathcal{S}_{S}^{*}\left(e^{z}\right)$ is of the form (1.1), then $\left|a_{6}-a_{2} a_{5}\right| \leq \frac{1}{6}$. This result is also sharp. Unfortunately, the sharp bound of $a_{6}$ has not been obtained. From (2.2), we can only obtain that

$$
\left|a_{6}\right| \leq \frac{1}{6}+\varepsilon, \quad \varepsilon=0.016 \ldots
$$

with an obvious conjecture that the exact value of the bound is equal to $1 / 6$.

Open Access This article is licensed under a Creative Commons Attribution 4.0 International License, which permits use, sharing, adaptation, distribution and reproduction in any medium or format, as long as you give appropriate credit to the original author(s) and the source, provide a link to the Creative Commons licence, and indicate if changes were made. The images or other third party material in this article are included in the article's Creative Commons licence, unless indicated otherwise in a credit line to the material. If material is not included in the article's Creative Commons licence and your intended use is not permitted by statutory regulation or exceeds the permitted use, you will need to obtain permission directly from the copyright holder. To view a copy of this licence, visit http:// creativecommons.org/licenses/by/4.0/.

\section{References}

1. Carlson, F.: Sur les coefficients d'une fonction bornée dans le cercle unité. Ark. Mat. Astr. Fys. 27A(1), 8 (1940)

2. Efraimidis, I.: A generalization of Livingston's coefficient inequalities for functions with positive real part. J. Math. Anal. Appl. 435(1), 369-379 (2016) 
3. Ganesh, K., Bharavi, S.R., Rajya, L.K.: Third Hankel determinant for a class of functions with respect to symmetric points associated with exponential function. WSEAS Trans. Math. 19, 13 (2020)

4. Obradović M., Tuneski N., Zaprawa P.: The sharp bound of the third Hankel determinant for univalent starlike functions. accepted for publication in Math.Bohem

5. Prokhorov, D.V., Szynal, J.: Inverse coefficients for $(\alpha, \beta)$-convex functions. Ann. Univ. Mariae CurieSkłodowska Sect. A 35, 125-143 (1981)

6. Zaprawa, P.: Initial logarithmic coefficients for functions starlike with respect to symmetric points. Bol. Soc. Mat. Mex. 27, 62 (2021)

7. Zaprawa, P., Obradović, M., Tuneski, N.: Third Hankel determinant for univalent starlike functions. Rev. R. Acad. Cienc. Exactas Fís. Nat. Ser. A Mat. RACSAM 115, 49 (2021)

Publisher's Note Springer Nature remains neutral with regard to jurisdictional claims in published maps and institutional affiliations. 